Research Article

\title{
Study on Cargo Products' Layout Optimization of Truck Railway Line
}

\author{
Jie Liu $\left(\mathbb{1},{ }^{1}\right.$ ShiWei He $\left(\mathbb{D},{ }^{2}\right.$ and Meng Zhang ${ }^{1}$ \\ ${ }^{1}$ Shan Dong Jiaotong University, Ji Nan City, Shan Dong Province 100044, China \\ ${ }^{2}$ Department of Transportation Management Engineering, School of Traffic and Transportation, Beijing Jiaotong University, \\ Beijing 100044, China \\ Correspondence should be addressed to Jie Liu; ajiede1986@163.com
}

Received 21 February 2020; Accepted 25 April 2020; Published 13 May 2020

Academic Editor: Elena Zaitseva

Copyright (c) 2020 Jie Liu et al. This is an open access article distributed under the Creative Commons Attribution License, which permits unrestricted use, distribution, and reproduction in any medium, provided the original work is properly cited.

\begin{abstract}
Cargo products' layout is one of the problems that needs an important decision in freight railroads. The purpose of optimization of the cargo products' layout on railway lines is to make a rational cargo products' plan to satisfy customers' diversified transport demands. In this paper, a service network is designed using the method of node splitting. Then, an optimized model is built for the products' layout problem on busy main railway lines. The immune clone-variable neighborhood algorithm is used to solve the model. Finally, a numerical example is given to verify the model and algorithm. The result shows that the model and algorithm are of good practical applicability.
\end{abstract}

\section{Introduction}

Busy main railway lines (BMRL) in China connect the important cities with the main economic development regions. There are great demands for both passenger and freight transportations in China. However, the contradiction between supply and demand of some busy main railway lines has been an imbalance for a long time. Some BMRL in China will release their capacity gradually with the large-scale construction of high-speed railways. The tense situation of transport capacity on BMRL will be relieved, which is beneficial for constructing a rational transportation products' plan to meet diversified transport demand. Therefore, it has both theoretical and practical significance to study on cargo products' layout optimization on BMRL.

Cargo products' layout is one of the problems that needs an important decision in freight railroads. Optimization of cargo products' layout on railway lines is to make a rational cargo products' plan to satisfy customers' diversified transport demands, which should consider the railway resources (stations, lines, and vehicles) and maximization of the profits for carriers. In short, the essence of optimization of cargo products' layout is to acquire optimal utilization of railway resources under the premise that the transport demand has been satisfied. Recently, lots of researchers study on this field or similar fields. Cargo products' layout is similar to the service network design. The interested reader of the network design problem should consult the surveys by Magnanti and Wong [1], Farvolden et al. [2-5], and Barnhart et al. [2, 6, 7]. Meantime, lots of researchers applied service network design to railway. Lulli et al. [8] presented a case study on freight railway transportation in Italy and highlighted the main features of the Italian situation and proposed a customized mathematical model to design the service network; Zhu et al. [9] combined blocking, makeup, and empty car distribution in an integrated dynamic model, and they built a 2-layer service network: one for block flows and the other for car flows. Crainic [10] provided the first example of a tactical planning model which was applied to rail freight transportation. Holmberg and Hellstrand [11] proposed a Lagrangean heuristic within a branch-andbound $(\mathrm{B} \& \mathrm{~B})$ framework to solve the uncapacitated network design problem with single origin and destination for each commodity, which can be used to model the rail freight 
transportation. Jeong et al. [12] focused on (part of) the European freight railway system modeled as a hub-and-spoke network. The scope of their model, an integer linear program, is to find the transport routes, the frequency of services, the number of cars composing each train, and the transportation volumes. Recently, Andersen and Christiansen [13] and Pedersen et al. [14] developed a strategically model to design the transportation services; their model included fleet-sizing considerations. Pedersen's constraints require the same number of assets entering and exiting each terminal, which are called design-balanced constraints. Yaghini and Akhavan [15] presented a review of MCND problem modeling, their applications in rail freight transportation planning, and solution methods which have been developed to solve them. In the field of cargo products, there are also lots of research achievements. Akhavan [16] used graph theory to construct a railway express transportation network and constructed a mathematical model by applying network flow theory; Qiang [17] proposed an integrated method of product design and train operation plan based on carrying capacity and designed the transport product with the view of passenger flow analysis; Li and Jiang [18] analyzed the connotations, principles, and procedures of the agile development of railway freight products, constructed a railway freight operation flow with the consideration of its characteristics, and proposed a development model based on QFD. Liu et al. [19] studied the optimized transportation products' layout from both tactical and strategic aspects and presented the optimization flow chart, respectively. Milan et al. [20] proposed measures and visions of railway passenger transport development, methodical process, and strategic questions for sustainable railway passenger transport and infrastructure. Merve and Yilmaz [21] introduced a new problem called the time-dependent hierarchical Chinese postman problem that aims to minimize the total travel time and formulated as a mixed integer linear programme. Oleksii et al. [22] developed a decision-making model in the first approximation in the part of the operation and development of the railway transport system. Zhang et al. [23] introduced the HL-RF algorithm in the field of structural engineering and built travel time reliability of the railway container chain evaluation model. Mustafa et al. [24] proposed the Chinese postman problem (CPP) to solve the inspection machine routing problem. Aldona et al. [25] evaluated the complexity of the railway transport activity and linkages with other activities by properly selecting the type of management and its specific theories of management application. Muhammed et al. $[26,27]$ proposed a new mathematical formulation to represent the postman problem with variable service costs and proposed two heuristics for the solution of the windy postman problem with variable service cost problem.

To summarize, most papers focused on the design of network, and its variables denote whether a train service should be open, and they do not consider all kinds of activities during the station, such as makeup, breakup, and drop-and-pull operations in the classification yard. In addition, cargo products' layout problem should obtain the cargo product plan based on the diversified transport demand and transport resources, for it is different from the service network design problem. Meantime, the research on cargo products mainly focused on the train operation plan design without considering cargo demand characteristics and capacity of marshalingbreaking-up of the classification station. In addition, since the transport demands affect the result of the cargo products' layout, demand with different features will need different services. However, the demand feature is ignored in the current research studies. So, this paper solves the cargo products' layout problem considering the transport demand feature and transport resources and uses the way of node splitting to describe the service operation procedures clearly on the classification station. Meantime, the optimized products' layout model on BMRL is built, and the immune clone-variable neighborhood algorithm is applied to solve the model.

We have organized the paper as follows: Section 2 presents a brief description of construction of the cargo product network. Section 3 introduces a mathematical model for the cargo products' layout problem. Section 4 proposes an algorithm for blocking the problem. In Section 5 , we summarize the experimental results of the proposed algorithm to solve the cargo products' layout problem in China Railways. Finally, the conclusion and future research directions are presented in Section 6.

\section{Cargo Product Network Construction}

As we know, it will increase difficulty to consider both the optimization of the cargo product service path and its start and end nodes. Thus, to reduce the problem size, the cargo products' alternative set is constructed based on OD demand characteristics, attributes of cargo products, development trend of regional economic, and some special limited factors. Then, the cargo product network is constructed, which is a network expression of operating plan. In the network, nodes are marshaling stations, loading and unloading stations, and freight stations, while an arc in the network denotes a service. Busy main railway lines in China are mostly operated in a double-line way but with different passing capacities on each direction. Hence, the cargo network should construct, respectively, for each direction. As is shown in Figure 1, it is the initial cargo product network of up direction. Figure 1(a) denotes the physical line, while Figure 1(b) represents the cargo products' network. Traditional cargo product in China can be divided into express product and regular product by traveling speed. With the change of freight market, Scheduled Trains Product can better meet the development of freight market for its highquality service. In this paper, Scheduled Trains Product (STP for short) is the product which is organized as passenger trains. That is to say, the Scheduled Trains Product is freight trains with scheduled station, scheduled train number, scheduled route, scheduled time, and scheduled price. So, in this paper, Scheduled Trains Product is considered as one kind of product. To describe the operation process of different products on the classification yard clearly, we used the way of split nodes to analyze different operation processes. As we know, lots of research studies applied the way of split nodes, such as Jacques (1990). However, in Jacques's research, the way of split nodes is used to deal with the transfer between different modes which cannot describe the 


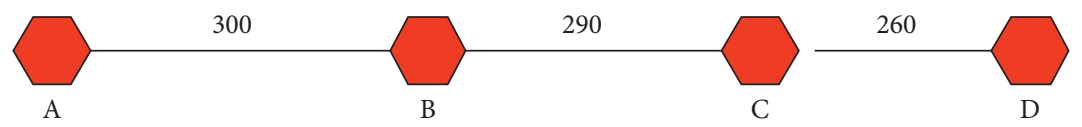

(a)

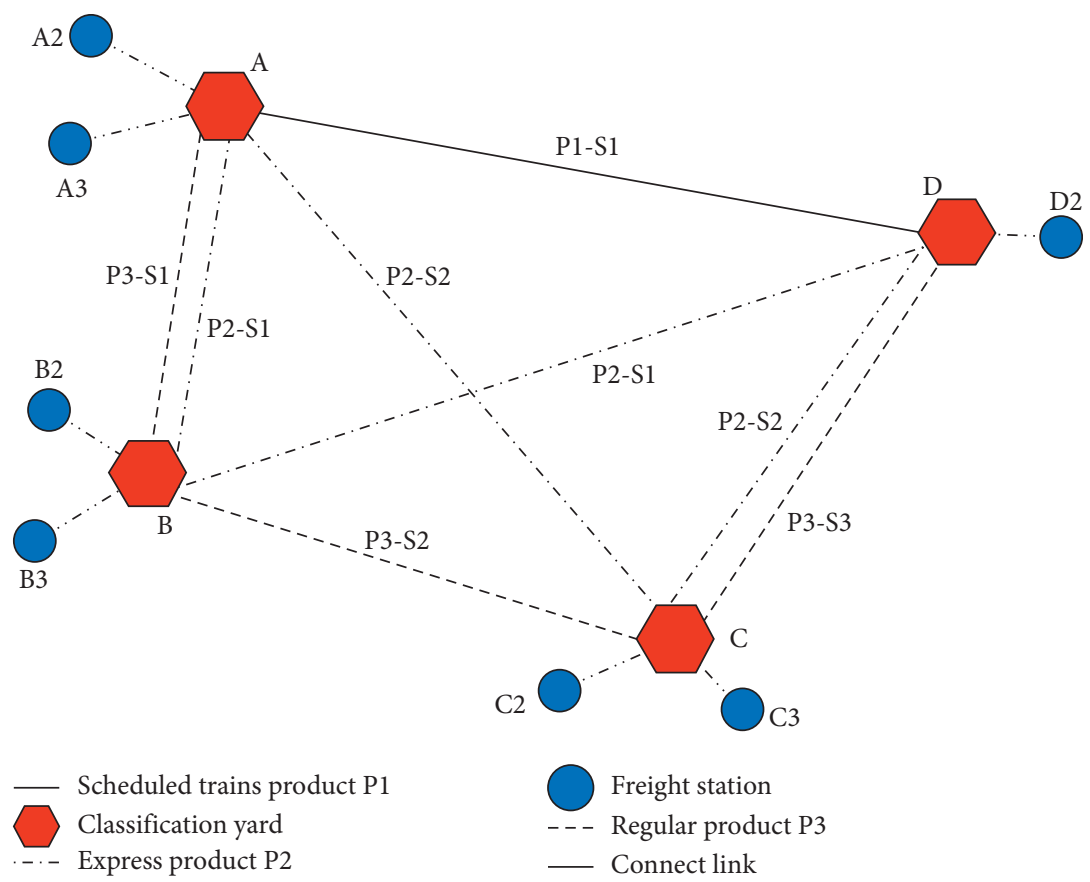

(b)

Figure 1: Cargo products' network for busy main railway lines.

operation process of different products on the station, so the new rules of split nodes are as follows.

If some cargo products do not need to be reclassified on the classification yard, nodes can be split according to the connected service number. In Figure 2, s1 and s2 are the service indices. The arcs between the split nodes denote transfer operations of service of cargo products.

Some kinds of cargo products need to be reclassified on the classification yard, nodes can be split into breakup and makeup operation nodes, and the passing time of arcs between these two nodes represents the average reclassifying time, as shown in Figure 3.

When reclassifying and transfer are both needed to be operated on some products, stations should be split using the two methods comprehensively, as shown in Figure 4.

In addition, to reduce the network size, freight demand in each region is represented by one virtual node. Otherwise, capacity constraints are also considered when building the model, so it is necessary to construct super-routes which can be built by adding supernodes that connect virtual nodes. Capacity and runtime on the super-route are set with a bigger value to ensure the super-route to be selected only when capacity on other routes is limited so that the demand quantity which is not satisfied in each OD can be obtained; both $\mathrm{A}^{\prime}-\mathrm{S}$ and $C^{\prime}-S$ in Figure 5 represent super-routes. Despite, the network size may be expanded using the way of splitting nodes, but nodes, which are split, are mostly marshaling stations that occupy limited quantity. Hence, the network size can be accepted. Through splitting nodes, transfer and reclassifying operations can be described clearly.

\section{The Mathematical Formulation}

The purpose of optimizing the layout of transportation products on BMRL is to acquire optimal utilization of the transport resources in order to form a rational freight product structure on the premise that diversified transportation demands of railway have been met. In addition, because of the railway's public welfare, it is vital to fully consider the transport demand attributes. Therefore, the paper classifies the demand into three layers by its characteristics: Priority Layer, Foundation Layer, and High Added-value Layer. Priority Layer mainly comprises materials related to people's livelihood, military supplies, emergency rescue, and disaster relief supplies. Foundation Layer includes some transportation of bulk materials which mainly serve region economy and do not belong to Priority Layer. In addition, Foundation Layer also includes some agriculture products' transportation demand such as cotton and grain. High Added-value Layer refers to transport demand of goods with high value that require high-quality services, and this layer mainly includes some electronic products, precision instruments, and food. 


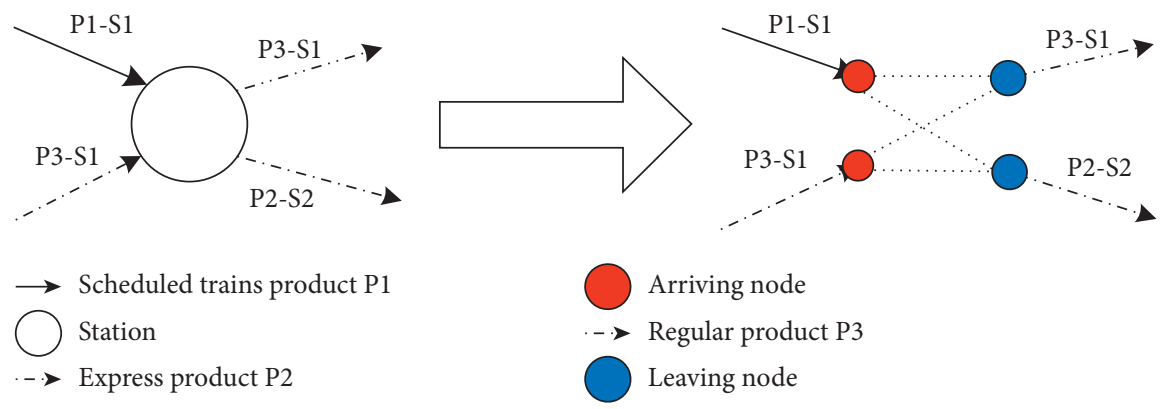

FIGURE 2: The way of node splitting for only having transfer operations.
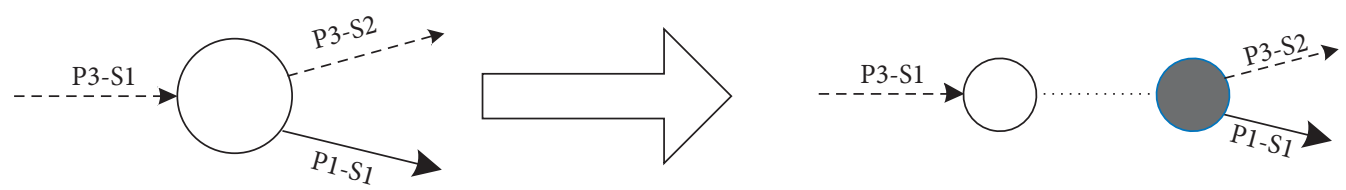

$\longrightarrow$ Scheduled trains product P1
Breakup operation node

- $>$ Regular product P3

Makeup operation node

FIgure 3: The way of node splitting for having sorting and classifying operations.
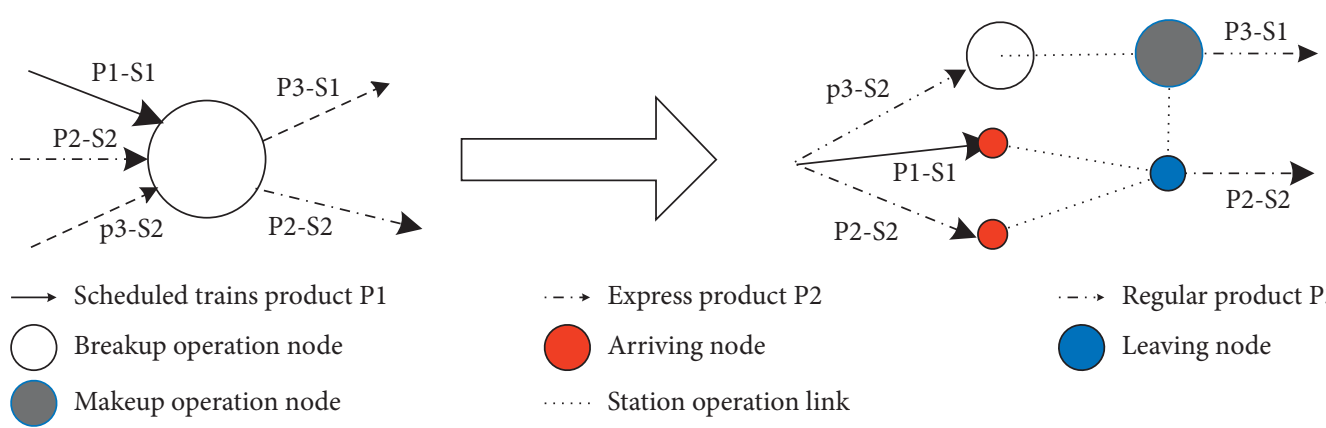

... Regular product P3

Leaving node

FIgURE 4: The way of splitting nodes for having reclassifying and transfer operations.

\subsection{Assumption}

(1) The freight demand is assumed to be steady without much fluctuation, and the transport deadline is determined by the customer's demand, also known as conditions

(2) No big changes happen on the stations' layout

(3) Constraints of mobile resources such as locomotives are enough to be used

(4) Cargo products' alternative set is given as the known condition

\subsection{Notations}

(1) Sets:

$\mathrm{G}(N, A)$ : the cargo products' network with node set $N$ and potential product set $A$.

$N_{c}$ : set of nodes with the breakup and makeup operation, $N_{c} \subset N$.
$A_{c}$ : set of arcs with the breakup and makeup operation, $A_{c} \subset A$.

$A_{s}$ : set of service arcs of cargo products, $A_{s} \subset A$. $A_{d}$ : set of arcs with transfer and drop or pull operation.

$A$ : arc set of physical lines.

$W$ : OD pair set.

$W^{u}$ : OD pair in the Priority Layer set, and $W^{u} \subset W$. $K$ : alternative set of cargo products where the product is $k$.

$\Gamma_{k}^{s}$ : set of service section with service $s$ of cargo product $k$, and $\vartheta \in \Gamma_{k}^{s}$ denotes one of the service sections.

$P_{w}$ : alternative route set of $w$ where one of the routes is $p$.

$S_{k}$ : service set of cargo products where $s$ denotes one kind of services.

(2) Variable parameters:

$T$ : decision-making period. 


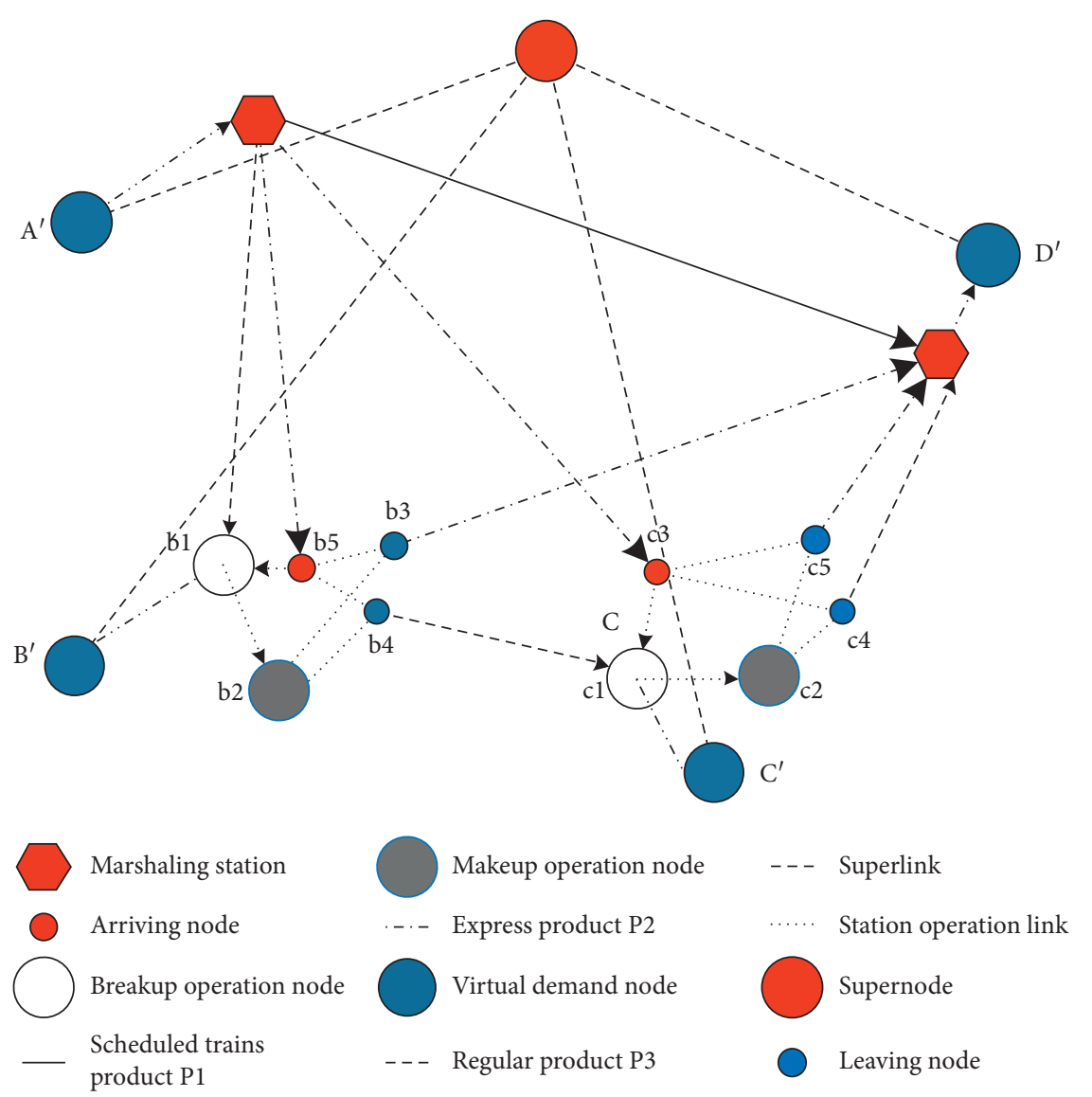

FIGURE 5: Cargo products' network after the node-splitting operation.

Cap $_{n}$ : capacity of node $n$ with the breakup and makeup operation, and $\mathrm{Cap}_{n}$ denotes breakup capacity where $n$ is the breakup operation node; otherwise $\mathrm{Cap}_{n}$ denotes makeup capacity.

$t_{a}$ : travel time of arc $a$. If $a \in A_{d}, t_{a}$ denotes the transfer time which can be calculated by $t_{a}=T / 2 f$ where $f$ is the frequency of the cargo product being waited; if $a \in A_{c}$, it denotes the average breakup and makeup operation time; if $a \in A_{s}$, it denotes the travel time of a service arc (unit: $h$ ).

$C_{a}$ : carrying capacity of $\operatorname{arc} a$ (unit: trains/T).

$d_{w}$ : the volume of traffic flow of OD pair $w$ (unit: car). $\pi_{w}: 0-1$ variables that indicate the relation between OD pair $w$ and the super-route. If OD pair $w$ can use the super-route, $\pi_{w}=1$ and 0 , otherwise.

$g_{w}$ : profit of OD pair $w$ (unit: RMB/car).

$L_{w}$ : travel distance of OD pair $w$.

$\tau_{a^{\prime}}^{s}: 0-1$ variables, indicating whether the service $s$ is passing section $a$.

$\varphi_{k}^{s}$ : fixed cost of services of product $k$.

$c_{a}$ : per unit cost of flow on $\operatorname{arc} a$.

$m_{k}$ : transport capacity of product $k$ for one service (unit: cars).

$l_{w}$ : freight transit period of OD pair $w$.

$\theta_{w}^{p, n}: 0-1$ variables; if node $n$ is included in the route $p$ of OD pair $w$, then $\theta_{w}^{p, n}=1$ and 0 , otherwise. $\beta_{w}^{p, k}: 0-1$ variables; if product $k$ is in the route $p$ of OD pair $w, \beta_{w}^{p, k}=1$ and 0 , otherwise. $\xi_{w}^{p, a}: 0-1$ variables; if arc $a$ is included in the service route $p\left(p \in P_{w}\right)$ of OD pair $w, \xi_{w}^{p, a}=1$ and 0 , otherwise.

$\gamma_{w}^{k}: 0-1$ variables; if transport demand of OD pair $w$ can be satisfied by cargo product $k, \gamma_{w}^{k}=1$ and 0 , otherwise.

$v_{k}^{s}$ : indicating the deducting coefficient of services of cargo product $k$ relative to the common product. $p_{u}^{w}$ : super-routes of OD pair $w$.

$\Psi_{k}$ : the maximum number of cargo products $k$.

(3) Decision variables:

$x_{w}^{p}$ : integer decision variables, represents flow on the service route $p$ of OD pair $w$, and $x_{w}^{u}$ is the flow on the superpath for OD pair $w$.

$\mu_{w}^{p}$ : 0 -1 variables. If route $p$ is selected for OD pair $w, \mu_{w}^{p}=1$ and 0 , otherwise, and $\mu_{w}^{u}$ if superpath is selected, $\mu_{w}^{u}=1$ and 0 , otherwise.

$f_{k}^{s}$ : operation frequency of service $s$ of cargo product $k$.

$\delta_{k}^{s}: 0-1$ variables. If service $s$ of product $k$ is operated during the decision-making period, $\delta_{k}^{s}=1$ and 0 , otherwise.

3.3. Formulation. Cargo product layout optimization model on the BMRL is to optimal utilizing transport resources and make cargo products' plan reasonably under the premise 
that the transport demand has been satisfied. It is essential to make products plan according to diversified transport demand with its level and attributes. The optimized model may be formulated as follows:

(1) Objective function:

The model takes maximizing the total transport profit as its objective:

(1) Revenue of transportation demand: $R=\sum_{w \in W}$ $\left(d_{w}-x_{w}^{u}\right) g_{w} L_{w}$.

(2) Fixed cost for operating: Cost $_{1}=$ $\sum_{k \in K} \sum_{s \in S_{k}} \varphi_{k}^{s} \delta_{k}^{s} f_{k}^{s}$.

(3) Transport and organization cost: Cost $_{2}=\sum_{w \in W} \sum_{a \in A} \sum_{p \in P_{w}} c_{a} \mu_{w}^{p} x_{w}^{p} \xi_{w}^{p, a} ;$ if $a \in A_{s}$, the function denotes transport cost. If $a \in A_{d} \cup A_{c}$, it denotes organization cost of the node.

The objective function for the model is

$$
\text { Min } Z=R-\text { Cost }_{1}-\text { Cost }_{2} \text {. }
$$

(2) Route-selected constraints:

$$
\sum_{p \in P_{w}} \mu_{w}^{p}=1, \quad \forall w \in W .
$$

Constraint (2) ensures that every OD pair can only be delivered by one route.

(3) Total transport demand amount:

$$
\sum_{p \in P_{w}\left\{p_{u}\right\}} \mu_{w}^{p} x_{w}^{p}+\pi_{w} \mu_{w}^{u} x_{w}^{u}=d_{w}, \quad \forall w \in W .
$$

For each OD pair, constraints (3) are balance equations; in addition, we set $\pi_{w}=0$ to ensure that the demand of Priority Layer must be met and 1, otherwise.

(4) Service capacity constraint of arcs:

$$
\sum_{w \in W} \sum_{p \in P_{w}} \mu_{w}^{p} x_{w}^{p} \zeta_{w}^{p, a} \leq \sum_{k} \sum_{s} \sum_{\vartheta \in \Gamma_{k}^{s}} \delta_{k}^{s} m_{k} \tau_{a}^{9} f_{k}^{s}, \quad \forall a \in A_{s} .
$$

Constraint (4) ensures the flow on the arc cannot be greater than the arc capacity.

(5) Marking-up and breakup capacity constraint of technical stations:

$$
\sum_{w \in W} \sum_{p \in P_{w}} \mu_{w}^{p} x_{w}^{p} \theta_{w}^{p, n} \leq \mathrm{Cap}_{n}, \quad \forall n \in N_{c} .
$$

For each technical station, constraint (6) prevents flow on the node be greater than the node capacity.

(6) Total amount of cargo products:

$$
\sum_{s \in S_{k}} \delta_{k}^{s} f_{k}^{s} \leq \Psi_{k}, \quad \forall k \in K .
$$

Constraint (6) is the total quantity constraint of cargo product $k$ and enforces the upper bound $\Psi_{k}$ for cargo product $k$.
(7) Physical capacity of the section constraint:

$$
\sum_{k} \sum_{s} v_{k}^{s} \delta_{k}^{s} f_{k}^{s} \tau_{a^{\prime}}^{s} \leq C_{a^{\prime}}, \quad \forall k \in K, a^{\prime} \in \tilde{A} .
$$

Constraint (7) ensures that, for each physical section, the number of services for physical section should be less than physical carrying capacity.

(8) Freight delivery period constraint:

$$
\sum_{a} \zeta_{w}^{p, a} t_{a} \leq l_{w}
$$

For each OD pair, constraint (9) ensures the travel time on route $p$ must be less than the freight transit period.

(9) Matching constraint between cargo products and demand:

$$
\mu_{w}^{p} \beta_{w}^{p, k} \leq M \gamma_{w}^{k}, \quad \forall w \in W, p \in P_{W}, k \in K .
$$

Constraint (10) represents that the product transported must match the transport demand.

(10) Logic constraint of variables:

$$
\begin{aligned}
& x_{w}^{p} \in Z^{+}, \\
& \mu_{w}^{p} \in\{0,1\}, \\
& \delta_{k}^{s} \in\{0,1\} .
\end{aligned}
$$

\section{Solution of the Cargo Product Layout Optimization Model}

This section introduces the proposed algorithm for solving the model. Cargo product layout optimization model is a mixed integer programming model, and because the formula of transfer time is a nonlinear expression, it has been shown as a NP-hard problem. It is hard to solve the problem by using traditional exact algorithms. Meantime, the problem also needs to be solved in a very short time. When the scale of the problem becomes larger, it is hard to obtain a feasible solution if using commercial software. Heuristic algorithm can obtain a feasible solution, even an optimal solution in a short time. Therefore, heuristic algorithm is used to solve the problem. And many research papers show that the immune clone algorithm is very useful to solve the integer programming problem.

According to analysis of the structure of the decision variable, the proposed solution method is based on immune clone algorithm (ICA). But, in the standard immune clone algorithm, the mutation probability will be reduced with the increase of iterative steps, making it possible to fall into local optimal solution. So, we use the combination of the immune clone algorithm and a variable neighborhood search algorithm to solve the problem. A variable neighborhood search algorithm can increase the diversity of solutions to jump out of local optimal. As the cargo product layout problem is chosen as integer decision variance, the combination of 


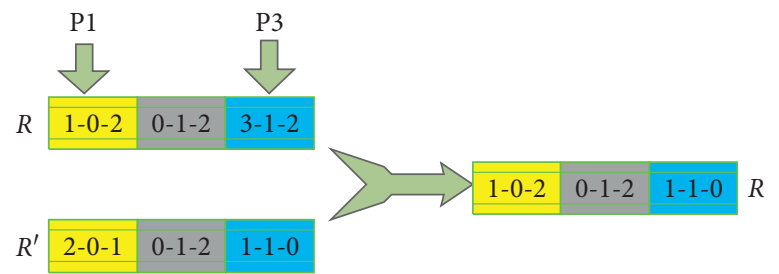

FIGURE 6: Update operators.

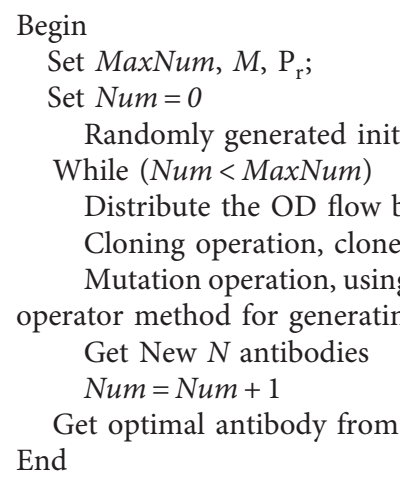

Algorithm 1: The pseudo-code of ICA-VNS.

immune clone algorithm and variable neighborhood search algorithm is used; this is called ICA-VNS algorithm. follows:

The solution steps of ICA-VNS algorithms are shown as

Step 1: the maximum number of iterations MaxNum is set, and Num is defined as the number of iterations. Creating the initial population and letting Num $=0$. Then, initial population of $N$ antibodies is generated randomly, which contains memory cell $M$ and remaining cells $P_{r}$. Integer encoding is adopted to solve this problem. The length of the antibody is the sum of the total number of services in the cargo product alternative set. Each antibody's range is from 0 to the maximum frequency of service, and 0 represents not open the service.

Step 2: transfer waiting time is calculated based on the frequency of service. Then, the kth shortest path between OD pairs is calculated by $A^{*}$ algorithm. At last, we can get $k$ service paths between each OD pair. If constraints (8) and (9) are not satisfied, then new antibodies are needed to be generated randomly.

Step 3: distributing the OD flow based on software CPLEX and letting Num $=0$. The objective function is defined as the antibody affinity function. It is in sequence carried out according to the degree of affinity. When Num $=0$ happens, antibodies with the first $n$ high-affinity values should be put into the memory cells $M$. When Num $>0$ happens, $N_{a}$ antibody affinity values are calculated and sequenced. If antibody affinity values are better than the ones in $M$, they are replaced. Then, antibodies that have lower affinity values in $P_{r}$ are replaced with antibodies that have higher affinity values in $N_{a}$.Then, the antibody population is cloned according to certain rules. $N_{i}=\operatorname{round}((\beta \times N) / i)$. Let $N_{i}$ be the number of antibody clones and $\beta$ be the clone factor. Round $(\cdot)$ is defined as the integer function and population size after cloning $N_{a}=\sum_{i} N_{i}$. If Num equals to MaxNum, turn to Step 6; otherwise, Step 5.

Step 4: mutation after cloning. Using variable neighborhood search algorithm can avoid falling into local optimal solutions and make it easier to get the global optimal solution.

Step 4-1: the more number of neighborhoods needed in a variable neighborhood search algorithm, the greater the probability of getting optimal solutions. The searching time will be increased dramatically with the increase in the number of neighborhoods. To ensure the speed and efficiency of solving, we always use two or three general methods for generating neighborhood. Considering the solution structure and characteristics, two methods will be adopted.

(1) Update operator: after the distribution operation, the frequency of services should be calculated, and then the updated antibody $R^{\prime}$ will be constituted. The method of updating operators is as follows. Either gene fragment in antibody $R$ is selected randomly and then compared with the antibody $R^{\prime}$ gene fragment, and antibody $R$ will be replaced by the one in antibody $R^{\prime}$ if it is different; otherwise, another gene fragment is selected randomly. As is shown in Figure 6, $R$ is the original antibody, and $R^{\prime}$ 
TABLE 1: Demand of OD pairs.

\begin{tabular}{|c|c|c|c|c|}
\hline Index & Origin & Destination & Volume (cars) & Deadline $(h)$ \\
\hline 1 & DSQ & $\mathrm{JZ}$ & 1 & 24 \\
\hline 2 & DSQ & NGL & 1 & 24 \\
\hline 3 & $\mathrm{HEB}$ & DSQ & 60 & 72 \\
\hline 4 & HEB & DSQ & 22 & 36 \\
\hline 5 & HEB & $\mathrm{JZ}$ & 262 & 96 \\
\hline 6 & HEB & $\mathrm{JZ}$ & 28 & 48 \\
\hline 7 & HEB & KY & 1 & 48 \\
\hline 8 & HEB & LY & 3 & 72 \\
\hline 9 & HEB & LS & 4 & 72 \\
\hline 10 & HEB & LS & 1 & 36 \\
\hline 11 & HEB & NGL & 23 & 96 \\
\hline 12 & HEB & SYX & 371 & 72 \\
\hline 13 & HEB & SYX & 25 & 36 \\
\hline 14 & HEB & SP & 14 & 48 \\
\hline 15 & HEB & SP & 1 & 24 \\
\hline 16 & HEB & SJT & 36 & 72 \\
\hline 17 & HEB & TLZ & 2 & 24 \\
\hline 18 & HEB & TLZ & 1 & 24 \\
\hline 19 & HEB & $\mathrm{TL}$ & 1 & 48 \\
\hline 20 & HEB & $\mathrm{CC}$ & 9 & 24 \\
\hline 21 & $\mathrm{JZ}$ & NGL & 1 & 24 \\
\hline 22 & LY & DSQ & 3 & 24 \\
\hline 23 & LY & $\mathrm{JZ}$ & 1 & 48 \\
\hline 24 & LY & LS & 80 & 24 \\
\hline 25 & LY & NGL & 1 & 48 \\
\hline 26 & LS & DSQ & 3 & 24 \\
\hline 27 & LS & $\mathrm{JZ}$ & 116 & 48 \\
\hline 28 & SP & DSQ & 46 & 48 \\
\hline 29 & SP & DSQ & 70 & 24 \\
\hline 30 & SP & $\mathrm{JZ}$ & 87 & 72 \\
\hline 31 & SP & $\mathrm{JZ}$ & 25 & 36 \\
\hline 32 & SP & LY & 2 & 48 \\
\hline 33 & SP & LS & 4 & 48 \\
\hline 34 & SP & NGL & 19 & 72 \\
\hline 35 & SP & NGL & 1 & 72 \\
\hline 36 & SP & SYX & 221 & 24 \\
\hline 37 & SP & SYX & 16 & 12 \\
\hline 38 & SP & SJT & 12 & 24 \\
\hline 39 & SP & $\mathrm{TL}$ & 1 & 24 \\
\hline 40 & SJT & DSQ & 10 & 24 \\
\hline 41 & SJT & $\mathrm{JZ}$ & 45 & 48 \\
\hline 42 & SJT & $\mathrm{JZ}$ & 9 & 24 \\
\hline 43 & SJT & LY & 1 & 24 \\
\hline 44 & SJT & LS & 14 & 24 \\
\hline 45 & SJT & NGL & 12 & 48 \\
\hline 46 & SJT & NGL & 1 & 24 \\
\hline 47 & TLZ & DSQ & 2 & 72 \\
\hline 48 & TLZ & $\mathrm{JZ}$ & 14 & 96 \\
\hline 49 & TLZ & LY & 1 & 48 \\
\hline 50 & TLZ & NGL & 1 & 96 \\
\hline 51 & TLZ & SYX & 15 & 48 \\
\hline 52 & TLZ & SP & 1 & 24 \\
\hline 53 & $\mathrm{TL}$ & DSQ & 5 & 24 \\
\hline 54 & $\mathrm{TL}$ & $\mathrm{JZ}$ & 9 & 48 \\
\hline 55 & $\mathrm{TL}$ & LY & 20 & 24 \\
\hline 56 & $\mathrm{TL}$ & LS & 5 & 24 \\
\hline 57 & $\mathrm{TL}$ & NGL & 1 & 48 \\
\hline 58 & $\mathrm{TL}$ & SYX & 11 & 24 \\
\hline 59 & $\mathrm{TL}$ & SJT & 29 & 24 \\
\hline 60 & $\mathrm{CCB}$ & DSQ & 97 & 48 \\
\hline
\end{tabular}

TABLE 1: Continued.

\begin{tabular}{lcccc}
\hline Index & Origin & Destination & Volume (cars) & Deadline $(h)$ \\
\hline 61 & CCB & LY & 1 & 48 \\
62 & CCB & LS & 1 & 48 \\
63 & CCB & NGL & 4 & 72 \\
64 & CCB & SYX & 141 & 48 \\
65 & CCB & SP & 13 & 24 \\
66 & CCB & SJT & 1 & 48 \\
67 & CCB & TL & 1 & 24 \\
68 & CCB & SP & 8 & 12 \\
\hline
\end{tabular}

is the new antibody. One gene fragment is selected randomly, such as the service decision scheme of product P3. It is clear that the service decision scheme between $R$ and $R^{\prime}$ is different for P3. So, gene fragment 2-1-2 in $R$ is updated to1-1-0 in $R^{\prime}$.

(2) Single-point mutation operator: randomly selected one point in antibody $R$ the singe point mutation is used to generate the new gene. These frequency values will be updated to any value from 0 to the maximum frequency value.

Step 4-2: a random number $r \in[1,2]$ is generated, and the mutation operation operators are selected according to the value of $r$. Let $N u m=N u m+1$. After the $N_{a}$ antibody carries out mutation operation, go to Step 6 if Num becomes MaxNum; otherwise, turn to Step 2.

Step 5: the optimal antibody is selected as an output from the memory cell $M$.

The pseudo-code of ICA-VNS is given in Algorithm 1.

\section{Case Study}

To verify the model and algorithm, this paper takes Ha-Da railway of China as a case to study the optimized cargo products' layout problems. Ha-Da railway is located in the north of China. According to the data analysis, because of the influence of transport capacity and cargo product, the railway demand mainly includes bulk material demand, and lots of high add-value demands be transported by motor carriers. With the construction of Ha-Da high-speed railway, lots of passenger trains on the existing railway line of Ha-Da will stop running. Therefore, transport capacity on the existing railway line of $\mathrm{Ha}-\mathrm{Da}$ will be relieved, which is beneficial to make the rational cargo products' plan to attract diversified transport demand, especially for attracting the high add-value demand. Part of transport demands and product attributes of the cargo product alternative set are shown in Tables 1 and 2 .

Through the survey, it is found that the makeup and breakup capacity of HEB is bottleneck. Therefore, in this case, we only consider the makeup and breakup capacity of HEB. According to the corresponding literature, we set the makeup capacity of HEB as 4,546 cars per day, and the breakup capacity of HEB is 5,200 cars per day. Meantime, as shown in the formulation, the carrying capacity of the 
TABLE 2: Service attributes of the cargo product alternative set.

\begin{tabular}{|c|c|c|c|c|c|}
\hline Index & Product type & Section & Carrying capacity per unit (car) & Travel time $(h)$ & Transport cost per unit (RMB/car) \\
\hline 1 & Scheduled Trains Product & HEB-CCB-SP & 50 & $5.02+2.58$ & $357.93+184.19$ \\
\hline 2 & Scheduled Trains Product & HEB-NGL & 50 & 13.11 & 1401.54 \\
\hline 3 & Scheduled Trains Product & $\begin{array}{c}\text { HEB-CCB- } \\
\text { SYX }\end{array}$ & 55 & $5.02+6.93$ & $357.93+493.77$ \\
\hline 4 & Scheduled Trains Product & HEB-LS-JZ & 50 & $8.74+3.96$ & $934.79+423.48$ \\
\hline 5 & Express product & HEB-KY-SJT & 55 & $6.23+1.64$ & $605.29+159.58$ \\
\hline 6 & Express product & HEB-CCB & 50 & 3.35 & 325.39 \\
\hline 7 & Express product & HEB-SP-SJT & 55 & $5.07+2.31$ & $492.83+224.98$ \\
\hline 8 & Express product & HEB-TL-SYX & 55 & $6.71+1.26$ & $652.02+122.33$ \\
\hline 9 & Express product & SP-SYX & 50 & 2.9 & 281.44 \\
\hline 10 & Express product & SJT-LS-JZ & 50 & $0.87+3.96$ & $84.95+384.98$ \\
\hline 11 & Express product & CCB-DLB & 50 & 9.76 & 948.73 \\
\hline 12 & Regular product & HEB-SYX & 65 & 14.24 & 529.73 \\
\hline 13 & Regular product & HEB-SJT & 65 & 15.74 & 535.41 \\
\hline 14 & Regular product & HEB-SP & 65 & 10.14 & 344.98 \\
\hline 15 & Regular product & $\begin{array}{l}\text { HEB-TLZ- } \\
\text { CCB }\end{array}$ & 65 & $3.51+3.18$ & $119.45+108.32$ \\
\hline 16 & Regular product & CCB-SP & 65 & 3.45 & 117.21 \\
\hline 17 & Regular product & SP-KY-SJT & 60 & $2.31+3.28$ & $78.72+111.7$ \\
\hline 18 & Regular product & SP-TL-SYX & 60 & $3.27+2.52$ & $111.37+85.63$ \\
\hline 19 & Regular product & KY-TL & 60 & 0.96 & 32.65 \\
\hline 20 & Regular product & SP-JZ & 60 & 15.27 & 519.37 \\
\hline 21 & Regular product & SJT-JZ & 60 & 9.67 & 328.95 \\
\hline 22 & Regular product & SJT-LY & 60 & 1.35 & 46.06 \\
\hline 23 & Regular product & SJT-LS & 60 & 1.75 & 59.46 \\
\hline 24 & Regular product & LS-DSQ & 60 & 2.19 & 74.36 \\
\hline 25 & Regular product & LY-JZ & 60 & 8.32 & 282.89 \\
\hline 26 & Regular product & DSQ-JZ & 60 & 5.74 & 195.13 \\
\hline 27 & Regular product & LS-JZ & 60 & 8.73 & 297.02 \\
\hline 28 & Regular product & JZ-NGL & 60 & 0.81 & 27.53 \\
\hline 29 & Regular product & LY-LS & 60 & 0.70 & 16.08 \\
\hline 30 & Regular product & SP-SJT & 60 & 5.59 & 197 \\
\hline 31 & Regular product & TL-SJT & 60 & 2.23 & 85.14 \\
\hline
\end{tabular}

section will affect the result of cargo products' layout plan. According to data analysis, the section from SP to SYX is also the bottleneck. The carrying capacity of this section is 74 trains per day. Other cost data needed in the example are summarized in Table 3. Otherwise, we assume decisionmaking period $T=24 \mathrm{~h}$, and assume the maximum number of products $\mathrm{P} 1, \mathrm{P} 2$, and $\mathrm{P} 3$ is 10,10 , and 60 , respectively.

Through lookup correlation literature, we find that the reduction coefficient for the express product is between 2 and 3. Therefore, we use 2.5 and 1.8 as the reduction coefficients for express product and Scheduled Trains Product, respectively. Using the data above, immune clone-variable neighborhood algorithm is used to solve the problem, and the maximum iteration steps MaxNum =100, the mutation probability $\beta=30 \%$, and the population $N=40$. The final result shows that the cost of the product layout plan for Ha$\mathrm{Da}$ railway is $1319686.69 \mathrm{RMB}$, and the iterative process of ICA-VNS algorithm and IC (immune clone) algorithm is shown in Figure 7; the ICA-VNS algorithm has better convergence speed than the IC algorithm. Otherwise, we can obtain the cargo products' layout plan. The result of the cargo products' layout plan is shown in Table 4.

The results show that it should operate 48 trains for regular products, 4 trains for express products, and 1 train
TABLE 3: Other cost values.

\begin{tabular}{lcc}
\hline Cost items & Unit & Cost value \\
\hline Arc cost for passing a hub & $\mathrm{RMB} / \mathrm{car}$ & 20 \\
Waiting cost & $\mathrm{RMB} / \mathrm{car}$ ' & 0.6 \\
The price for railway & $\mathrm{RMB} / \mathrm{car}^{*}$ kilometers & 0.19 \\
Fixed service cost for P1 & $\mathrm{RMB} /$ service & 10,000 \\
Fixed service cost for P2 & $\mathrm{RMB} /$ service & 5,000 \\
Fixed service cost for P3 & $\mathrm{RMB} /$ service & 3,000 \\
Transport cost for superpath & $\mathrm{RMB} /$ cars & 100,000 \\
\hline
\end{tabular}

for Scheduled Trains Product. It is demonstrated that the main advantage of railway is still mass freight transport. At the same time, some trains for rapid products and train products are also operated when the capacity is relatively abundant according to the customers' demands. In addition, through the flow distribution result, it shows that the lines' capacity is no longer the bottleneck which restricts the railway freight transportation, but the nodes' capacity has been new restrictive factors. It is also illustrated that it is necessary to reconstruct and expand the hubs to further optimize the transportation products to improve hubs' breakup and marshaling capacity so that the transport market demand will be satisfied and the transportation efficiency will be improved. 


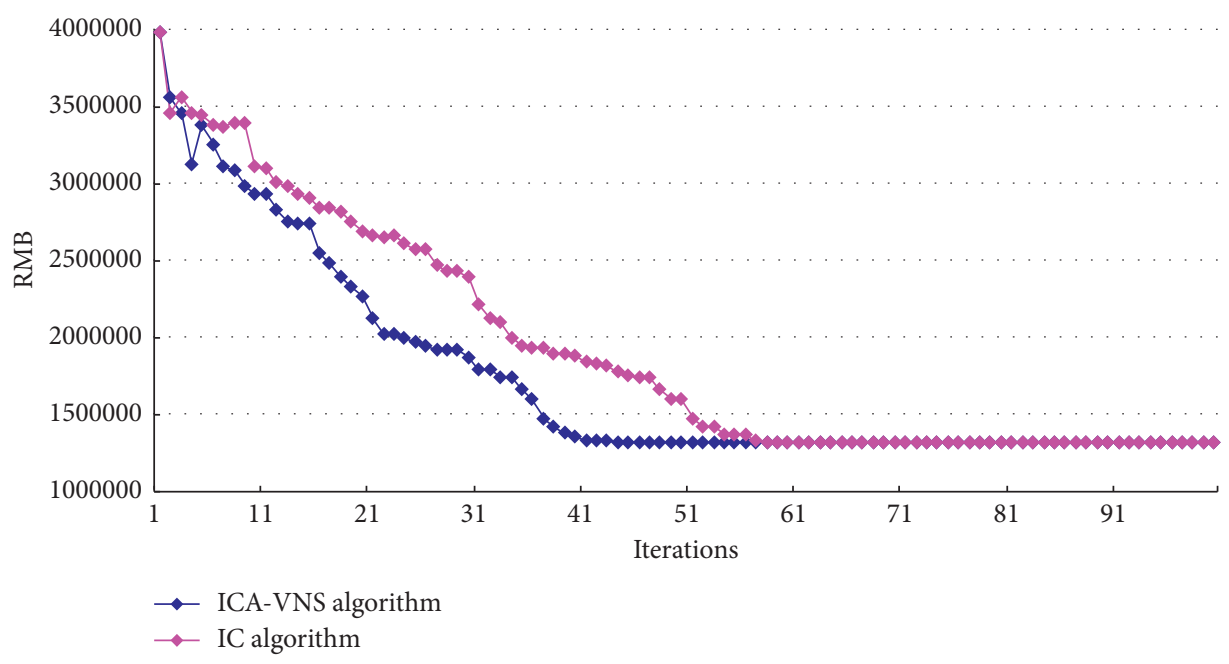

Figure 7: The iterative process of ICA-VNS algorithm and IC algorithm.

TABLE 4: Operation results of service products.

\begin{tabular}{lccc}
\hline Product code & Product type & Product index & Section \\
\hline P1 & Scheduled Trains Product & 4 & HEB-LS-JZ \\
P2 & Express product & 9 & SP-SYX \\
P2 & Express product & 10 & SJT-LS-JZ \\
P2 & Express product & 11 & CCB-DLB \\
P3 & Regular product & 12 & HEB-SYX \\
P3 & Regular product & 13 & HEB-SJT \\
P3 & Regular product & 14 & HEB-SP \\
P3 & Regular product & 15 & 2 \\
P3 & Regular product & 16 & HEB-TLZ-CCB \\
P3 & Regular product & 17 & CCB-SP \\
P3 & Regular product & 18 & SP-KY-SJT \\
P3 & Regular product & 20 & SP-TL-SYX \\
P3 & Regular product & 21 & SP-JZ \\
P3 & Regular product & 22 & SJT-JZ \\
P3 & Regular product & 23 & SJT-LY \\
P3 & Regular product & SJT-LS \\
P3 & Regular product & 24 & 10 \\
P3 & Regular product & 26 & 3 \\
P3 & Regular product & 27 & 1 \\
P3 & Regular product & 28 & 1 \\
P3 & Regular product & 29 & 6 \\
P3 & Regular product & 30 & 6 \\
& & 31 & 1 \\
& & LY-JZ
\end{tabular}

\section{Conclusion and Future Research}

Optimized transportation products' layout on railway lines refers to making a rational organization and operation of transportation products considering the current railway resources and the customers' transport demands at all levels to get reasonable product mix, operation ratio, and to maximize the profits of the operation department. The paper first constructs a service network of main busy lines which can effectively show the hub's transfer, sorting, and classifying operations. Then, an optimized model is suggested for the products' layout on BMRL, and the immune clonal-variable neighborhood algorithm is used to solve the model. Finally, an example is given to prove the effectiveness of the model and algorithm. However, the model put forward in the paper is built based on the assumption that the station layout is given, so it needs to study the problem on how to optimize the products' layout on BMRL further combined with the stations' layout.

\section{Data Availability}

The data in this paper are enough to verify the model and algorithm.

\section{Conflicts of Interest}

The authors declare that they have no conflicts of interest. 


\section{Acknowledgments}

This work was supported by Shandong Jiaotong University Doctoral Fund (no.BS201902020), Key Research, National Key R\&D Program of China (2018YFB1201402), Research Project of China Railway Corporation (P2018X003 and P2019X004), and Development Program of Shandong Province (2017GGX50110).

\section{References}

[1] T. L. Magnanti and R. T. Wong, "Network design and transportation planning: models and algorithms," Transportation Science, vol. 8, no. 1, pp. 16-55, 1984.

[2] J. M. Farvolden and W. B. Powell, "Subgradient methods for the service network design problem," Transportation Science, vol. 28, no. 3, pp. 256-272, 1994.

[3] C.-C. Lin and S.-H. Chen, "An integral constrained generalized hub-and-spoke network design problem," Transportation Research Part E: Logistics and Transportation Review, vol. 44, no. 6, pp. 986-1003, 2008.

[4] C.-C. Lin and S.-H. Chen, "The hierarchical network design problem for time-definite express common carriers," Transportation Research Part B: Methodological, vol. 38, no. 3, pp. 271-283, 2003.

[5] M. Minoux, "Networks synthesis and optimum network design problems: models, solution methods and applications," Networks, vol. 19, no. 3, pp. 313-360, 1989.

[6] C. Barnhart, A. Armacost, and K. Ware, "Composite variable formulations for express shipment service network design," Transportation Science, vol. 36, no. 1, pp. 1-20, 2002.

[7] T. G. Crainic, "Service design models for rail intermodel transportation," Lecture Notes in Economics and Mathematical Systems, vol. 619, pp. 53-67, 2009.

[8] G. Lulli, U. Pietropaoli, and N. Ricciardi, "Service network design for freight railway transportation: the Italian case," Journal of the Operational Research Society, vol. 62, no. 12, pp. 2107-2119, 2011

[9] E. Zhu, T. G. Crainic, and M. Gendreau, Integrated Service Network Design for Freight Transportation. Working Paper, CIRRELT: Interuniversity Research Centre on Enterprise Networks, Logistics and Transportation, Montreal, Canada, 2009.

[10] T. G. Crainic, "Service network design in freight transportation," European Journal of Operational Research, vol. 122, no. 2, pp. 272-288, 2000.

[11] K. Holmberg and J. Hellstrand, "Solving the uncapacitated network design problem by a Lagrangean heuristic and branch-and-bound," Operations Research, vol. 46, no. 2, pp. 247-259, 1998.

[12] S.-J. Jeong, C.-G. Lee, and J. H. Bookbinder, "The European freight railway system as a hub-and-spoke network," Transportation Research Part A: Policy and Practice, vol. 41, no. 6, pp. 523-536, 2007.

[13] J. Andersen and M. Christiansen, "Designing new European rail freight services," Journal of the Operational Research Society, vol. 60, no. 3, pp. 348-360, 2009.

[14] M. B. Pedersen, T. G. Crainic, and O. B. G. Madsen, "Models and tabu search metaheuristics for service network design with asset-balance requirements," Transportation Science, vol. 43, no. 2, pp. 158-177, 2009.

[15] M. Yaghini and R. Akhavan, "Multicommodity network design problem in rail freight transportation planning,"
Procedia-Social and Behavioral Sciences, vol. 43, pp. 728-739, 2012.

[16] L. Akhavan, "Design of railway express transportation products with dedicated lines for passenger and cargo transportation," Logistic Technology, vol. 31, no. 1, pp. 86-88, 2012.

[17] L. X. Qiang, "Research on transportation product design and train operation at same dedicated passenger railway line," Journal of Beijing Jiaotong University, vol. 32, no. 3, pp. 11-20, 2008.

[18] H. Y. Li and X. Jiang, "Study on the agile development of railway freight products," Logistic Technology, vol. 28, no. 12, pp. 27-29, 2009.

[19] J. J. Liu, H. D. Li, and S. W. He, "Optimized research on railway transportation products based on the condition of passenger and cargo separation," Journal of Comprehensive Transportation, vol. 3, pp. 73-76, 2011.

[20] D. Milan, C. Lukáš, and G. Jozef, "Methodical process for innovative management of the sustainable railway passenger transport," Transportation Research Procedia, vol. 44, pp. 305-312, 2020.

[21] K. R. Merve and M. Yilmaz, "A time-dependent hierarchical Chinese postman problem," Central European Journal of Operations Research, vol. 28, no. 1, pp. 337-366, 2018.

[22] K. Oleksii, K. Yevgeniia, and K. Oleksandr, "Improvement of the railway transport system by increasing the level of goaloriented activity," Procedia Computer Science, vol. 149, pp. 415-421, 2019.

[23] R. Zhang, L. Li, and W. Jian, "Reliability analysis on railway transport chain," International Journal of Transportation Science and Technology, vol. 8, no. 2, pp. 192-201, 2019.

[24] Y. Mustafa, K. Merve, and Y. Hamid, "Chinese postman problem approach for a large-scale conventional rail network in Turkey," Tehnicki vjesnik-Technical Gazette, vol. 24, no. 5, pp. 1471-1477, 2017.

[25] K. Aldona, S. Gintaras, and M. Asta, "Analysis of application management theories and methods for developing railway transport," Procedia Engineering, vol. 187, pp. 173-184, 2017.

[26] E. K. Muhammed and Y. Mustafa, "Chinese and windy postman problem with variable service costs," Soft Computing, vol. 23, pp. 7359-7373, 2019.

[27] B. H. Wang, "Research on multi-modal express shipment network resource planning optimization," Doctoral thesis, Beijing Jiaotong University, Beijing, China, 2010. 